\title{
Estructura, composición y diversidad arbórea y arbustiva en tres condiciones de manejo forestal de Ixtlán de Juárez, Oaxaca
}

\section{Tree and shrub structure composition and diversity in three forest management conditions in Ixtlan de Juarez, Oaxaca}

\author{
Vicente Florencio Vásquez-Cortez', Ricardo Clark-Tapia', Filemón Manzano-Méndez!' \\ Gabriel González-Adamel y Victor Aguirre-Hidalgo'
}

Universidad de la Sierra Juárez. Instituto de Estudios * Autor de correspondencia. victor@.unsij.edu.mx Ambientales. Ixtlán de Juárez, Oaxaca, México.

\section{RESUMEN}

Las prácticas forestales implican una serie de actividades que pueden generar impactos negativos en la dinámica natural de este ecosistema. El objetivo de este estudio fue evaluar el efecto del manejo forestal en la estructura, composición y diversidad arbórea y arbustiva en Ixtlán de Juárez, Oaxaca. Para ello se establecieron 15 unidades de muestreo distribuidas en tres condiciones de manejo: franjas no aprovechadas (FNA), bordes (B) y franjas aprovechadas (FA) establecidos en bosques con tratamiento de matarrasa. Se describen los cambios en estructura, composicón florística y diversidad de los estratos de árboles y arbustos. Durante el periodo de estudio se identificaron 27 especies vegetales distribuidas en nueve familias. Las especies más frecuentes fueron: Quercus crassifolia Humb \& Bonpl., Pinus psendostrobus Lindl. y Quercus laurina Humb. \& Bonpl. Las curvas de acumulación de especies mostraron que las FNA tuvieron un mayor número de especies que las otras dos áreas. La estructura, estimada a partir de los índices de valor de importancia (IVI) y de valor forestal (IVF), estuvo asociada a la presencia de las tres especies previamente mencionadas en las tres condiciones. La categoría fustal fue la dominante en FNA y B; mientras que en FA las categorías dominantes fueron las de latizal y brinzal. Los valores de riqueza fueron bajos en FNA, B, FA, pero con valores altos de equidad. El valor de dominancia fue solamente alto en FA; B y FA fueron los más semejantes entre sí. Se concluye que las prácticas silviculturales promueven la conformación de un bosque coetáneo con dominancia de pinos. Este patrón es menos severo dada la presencia de las FNA, las cuales podrían limitar la pérdida de la diversidad arbórea y arbustiva.

PALABRAS ClAVE: curvas especie-área, diversidad de especies, riqueza florística, perfil vertical, perfil horizontal.

\section{ABSTRACT}

Forest activities play a critical role in the natural dynamics of this ecosystem. The aim of this work was to evaluate the effect in structure composition and forest and shrub diversity of the forest management practices applied in Ixtlán de Juárez, Oaxaca. We established 15 sampling areas distributed among three silvicultural treatments: not harvested strips (FNA), edges (B), and harvested strips (FA) established in a strip clearcut timber harvesting forest. Changes in structure, floristic composition and diversity of trees and shrubs strata are described. Twenty-seven species and nine botanical families were identified, being the most frequent Quercus crassifolia Humb \& Bonpl., Pinus psendostrobus Lindl. and Quercus laurina Humb. \& Bonpl. Accumulation species-area curves were estimated, showing that the FNA condition had the highest number of species. Through the importance value index (IVI) and forest value index (IVF) it was found that structural value varied for each species, size class (stems, stalks and saplings), and condition (FNA, B and F). Higher values on IVI and IVF were consistently obtained by the three previously mentioned species in the three conditions. However, stems represented the dominant structure in FNA and B while stalks and saplings were dominant in FA. According to diversity indices, richness was low, but evenness was high in the three conditions (FNA, B, FA). Also, dominance was high in FA. B and FA condition were the most similar between them. We concluded that during the period analyzed the silvicultural practices promote an even-aged forest. However, this pattern is less intense because of FNA presence which could limit arboreal and shrub diversity loss.

KEYWORDS: floristic richness, species diversity, species area curves, vertical profile, horizontal profile. 


\section{INTRODUCCIÓN}

Los bosques templados son áreas importantes para la diversidad biológica, ya que proveen de una serie de bienes y servicios ecosistémicos, y son eje de desarrollo económico importante a escala local, nacional e internacional (Valencia-Avalos y Nixon, 2004; Challenger, 1998). En México, son una base económica importante debido a su producción maderable, sobre todo en los estados de Durango, Guerrero, Jalisco, Michoacán y Oaxaca (Jiménez, Aguirre y Kramer, 2001). A escala nacional se estima una producción de aproximadamente 5501085 metros cúbicos-rollo ( $\mathrm{m}^{3} \mathrm{r}$; Secretaria de Medio Ambiente y Recursos Naturales, 2012), de los cuales el estado de Oaxaca aporta alrededor de $284 \times 10^{6} \mathrm{~m}^{3} \mathrm{r}$ (Comisión Nacional Forestal, 2008).

En particular, el predio comunal de Ixtlán de Juárez tiene una producción anual de $32893 \mathrm{~m}^{3} \mathrm{r}$ (Servicios Técnicos Forestales de Ixtlán de Juárez [STF], 2014). Los bosques comerciales de esta región son de segundo crecimiento y están conformados principalmente por pinos y encinos de diferentes clases de edades (e.g. son irregulares e incoetáneos), estos rodales han estado bajo aprovechamiento maderable por más de siete décadas (Castellanos-Bolaños, Treviño-Garza, Aguirre-Calderón, Jiménez-Pérez y Velázquez-Martínez 2010; Técnica Informática Aplicada S.A. [TIASA], 2003). Los bosques de esta región, tradicionalmente se han repoblado con cortas de regeneración con el método de selección grupal en franjas alternas, el cual consiste en aplicar cortas a matarrasa (llamadas también cortas totales) en una franja de ancho específico, pero de longitud dependiente de las condiciones del terreno, con franjas contiguas intactas (STF, 2014).

Esta técnica se aplica en todo el rodal seleccionado de manera cíclica. En el siguiente ciclo de aprovechamiento se vuelve a aplicar el tratamiento de matarrasa, pero esta vez en las franjas no aprovechadas. Este segundo ciclo se realiza después de que se inició el proceso de reforestación en las franjas del primer ciclo, es decir, aproximadamente 30 años después del primer corte (Hawley y Smith, 1982). Una consecuencia negativa que genera este tipo de manejo es la modificación drástica, de la estructura y composición espacial de la vegetación en un lapso muy breve, al provocar cambios significativos en las condiciones bióticas y abióticas del ecosistema (e.g. Clark-Tapia, et al., 2017; Del Castillo, Pérez de la Rosa, Vargas y Rivera, 2004; Fregoso, Velásquez, Brocco y Cortéz, 2001; Murcia, 1995), lo cual puede generar una drástica disminución de la diversidad biológica (e.g. Corral-Rivas, Jiménez-Pérez, AguirreCalderón y Corral-Rivas, 2005).

Es necesario conocer las características de la estructura arbórea y arbustiva de las zonas silvícolas de Ixtlán de Juárez, zona que está incluida en el programa de Regiones Terrestres Prioritarias-130 (Sierras del norte de OaxacaMixe) (Arriaga et al., 2000), como una base importante al momento de especificar un método de aprovechamiento maderable que permita reducir el impacto generado. Para que lo anterior sea posible, se requiere información de este tipo de ecosistema, no solo de las zonas directamente bajo aprovechamiento maderable, sino también de las áreas naturales adyacentes a las áreas aprovechadas y de las zonas de transición (bordes). Con base en lo anterior, el presente trabajo busca conocer el efecto que genera el manejo silvícola sobre la diversidad biológica en zonas forestales de Ixtlán de Juárez, Oaxaca, para lo cual se estimaron índices estructurales, de diversidad, distribución y abundancia de especies arbóreas y arbustivas en zonas bajo aprovechamiento forestal.

\section{OBJETIVOS}

El objetivo principal del estudio fue evaluar la estructura, composición y diversidad de las especies arbóreas y arbustivas en tres condiciones de manejo: franjas no aprovechadas, franjas aprovechadas y bordes, ubicadas en el bosque de Ixtlán de Juárez, Oaxaca.

\section{MATERIALES Y MÉTODOS}

\section{Área de estudio}

El presente estudio se realizó en la zona de aprovechamiento forestal de Ixtlán de Juárez. La zona forma parte de la región denominada Sierra Juárez y está ubicada entre las coordenadas $17^{\circ} 21^{\prime} 24^{\prime \prime}$ y $17^{\circ} 21^{\prime} 08^{\prime \prime} \mathrm{N}$; 
$96^{\circ} 28^{\prime} 59^{\prime \prime}$ y $96^{\circ} 28^{\prime} 26^{\prime \prime} \mathrm{W}$, a una elevación promedio de $2500 \mathrm{~m}$ snm y con pendientes de $35 \%$. (Fig. 1). La superficie total del predio es de aproximadamente 19492.44 ha, de la cual 45\% (8 835.44 ha) es bosque comercial para madera de pino principalmente y en menor cantidad de encino.

Ixtlán de Juárez se caracteriza por su relieve geográfico heterogéneo, con laderas y pendientes que fluctúan en un intervalo de $40 \%$ a $60 \%$ y una altitud que varía entre los $1500 \mathrm{~m}$ y los $3200 \mathrm{~m}$ snm. El clima es templado subhúmedo con lluvias en verano C(w"1)(w)b(i')g (Clark-Tapia, SuárezMota, Venegas-Barrera y Velasco-Hipólito, 2016). Pertenece a la Región Hidrológica No. 28 denominada "Papaloapan" y se caracteriza por tener numerosos escurrimientos sinuosos que sirven de recarga al río conocido como "Shoo-Beeto" el cual desemboca en el Río Grande (Alfonso-Corrado et al., 2016). La precipitación media anual oscila entre $900 \mathrm{~mm}$ y $1700 \mathrm{~mm}$, la temperatura media anual es de $18{ }^{\circ} \mathrm{C}$ aproximadamente (Clark-Tapia, Suárez-Mota, Matías-Nolasco y Velasco-Hipólito, 2016). El tipo de suelo es luvisol (l) con textura media limosa, además presenta acumulación de arcilla en el subsuelo (Clark-Tapia, Rodríguez-Rivera, Ramos-Morales y Velasco-Hipólito, 2016).

La vegetación está constituida por bosque de pinoencino y vegetación secundaria derivada del bosque de pino-encino (Clark-Tapia, Sandoval-García, Ramos-Morales y Rodríguez-Rivera, 2016). El número de especies vegetales es de aproximadamente 6000, de las cuales destacan 13 especies de Pinus (Del Castillo, Pérez de la Rosa, Vargas y Rivera, 2004) y 23 de Quercus (Valencia-Avalos y Nixon, 2004), además de otros géneros como Arbutus, Alnus, Prunus, Fraxinus y Litsea (Ruiz-Aquino et al., 2014).

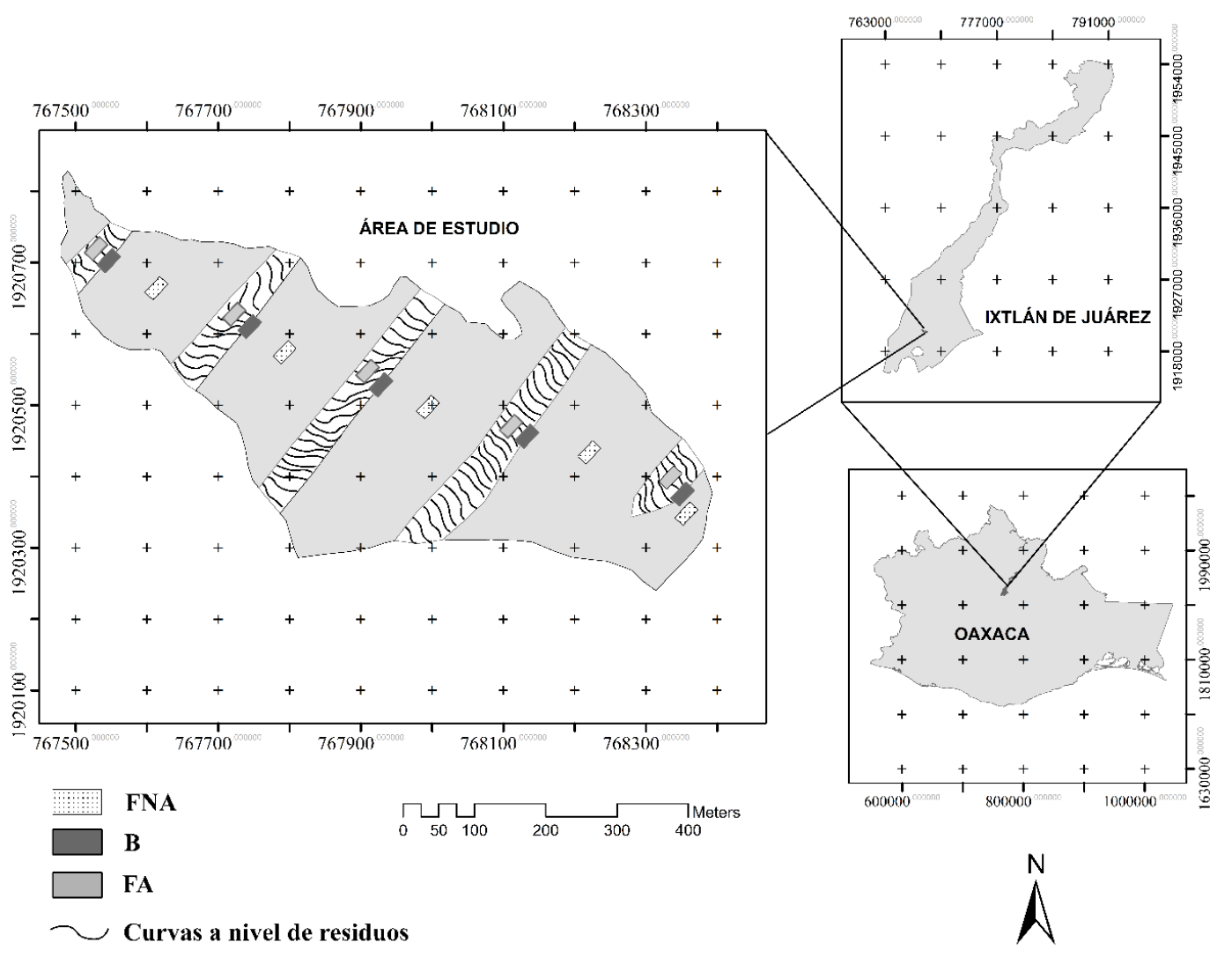

FIGURA 1. Ubicación geográfica de Ixtlán de Juárez y del área de estudio.

FNA = franjas no aprovechadas, $\mathrm{B}$ = bordes y FA = franjas aprovechadas, del periodo 2011. 


\section{Identificación de especies}

Dentro del área de estudio se establecieron tres transectos orientados de manera perpendicular a las franjas de manejo forestal; dos fueron ubicados a diez metros de los bordes de la zona de manejo, el tercer transecto se ubicó en la parte media del área de manejo, a una distancia equidistante a los dos primeros. Se colectaron manualmente estructuras tanto vegetativas como reproductivas de árboles y arbustos de individuos ubicados a una distancia máxima de un metro del transecto. El material colectado se herborizó de manera tradicional y se depositaron en una secadora, para posteriormente identificarlas a partir de las claves dicotómicas de Luteyn et al. (1995); Farjon, Pérez de la Rosa y Styles (1997); McVaugh, (1984); Rzedowski, (2005) y Zavala (2003). El material en el que hubo duda en la identificación fue corroborado a partir de ejemplares del Herbario Nacional de México (MEXU) de la Universidad Nacional Autónoma de México.

\section{Diseño de muestreo}

Las franjas estudiadas fueron aprovechadas en 2010 de con el plan elaborado en 2005 (TIASA, 2003). En esta zona se ubicó un transecto en la parte media del área de manejo forestal (Fig. 1) y se establecieron cinco unidades de muestreo $(\mathrm{UM})$ de $10 \mathrm{~m} \times 20 \mathrm{~m}\left(200 \mathrm{~m}^{2}\right)$ en cada condición de manejo [franjas no aprovechadas (FNA), bordes (B) y franjas aprovechadas (FA)]. Cada UM se dividió en cuadrantes de $10 \mathrm{~m} \times 10 \mathrm{~m}$, los cuales fueron denominados subunidades de muestreo (SUM). En cada SUM se midieron todos los fustales con diámetro normal igual o mayor a $2.5 \mathrm{~cm}$. Para medir los latizales, individuos con diámetro normal menor a $2.5 \mathrm{~cm}$ y altura mayor a $1.3 \mathrm{~m}$, se trazaron de manera aleatoria cuatro cuadros de $5 \mathrm{~m} \times 5 \mathrm{~m}$. Para medir los brinzales, individuos con altura menor o igual a $1.3 \mathrm{~m}$, se establecieron dentro de los cuadros de $5 \mathrm{~m}$ $\times 5 \mathrm{~m}$ dos cuadros de $1 \mathrm{~m} \times 1 \mathrm{~m}$ (modificado de ValdezHernández, 2002). Las variables consideradas fueron altura total, diámetro a la altura del pecho (DAP) y cobertura (proyección de las copas en los ejes $x$ y $y$ ).

\section{Análisis de la información}

A través del programa PRIMER-E Ltd. (Clarke y Gorley, 2006) se elaboraron las curvas especie-área, se calcularon los índices de riqueza de especies de Margalef (1977), así como los índices de diversidad: de Shannon-Weiner $\left(\mathrm{H}^{\prime}\right)$, de Simpson (S) y de equidad (E). Además, para estimar la similitud entre sitios, se calcularon los índices de Sorensen $\left(\mathrm{C}_{\mathrm{s}}\right)$ y de Jaccard $\left(\mathrm{C}_{\mathrm{j}}\right)$, los cuales describen la diferencia espacial y las diferencias en riqueza de especies entre las comunidades (Koleff, Gaston y Lennon, 2003) (Tabla 1). $\mathrm{El}$ analisis de varianza (Anova) y la prueba de comparación de medias (Tukey $p<0.05$ ) para las tres condiciones de manejo (FNA, B y FA) fue realizado en Infostat (Infostat, 2008). Se aplicaron dos indices estructurales [índice de valor de importancia (IVI) e índice de valor forestal (IVF)], para jerarquizar la dominancia y evaluar la importancia de las especies arbóreas y arbustivas. Mediante el IVF, se determinó la estructura de la vegetación para las tres condiciones (FNA, B, FA) y para tres estados de desarrollo (fustal, latizal y brinzal).

El IVI, desarrollado por Curtis y McIntosh (1951) y aplicado por Corella et al., (2001), se utilizó para identificar la especie dominante, en términos de densidad y frecuencia:

$$
\begin{gathered}
\mathrm{IVI}=((\text { Dominancia relativa }+ \text { Densidad relativa } \\
+ \text { Frecuencia relativa }) / 3)
\end{gathered}
$$

La estimación del IVF se realizó para considerar la influencia de la altura de todos los individuos medidos en el rodal, como la densidad de cobertura del dosel (Corella et al., 2001).

$$
\begin{array}{r}
\mathrm{IVF}=((\text { Diámetro relativo }+ \text { Altura relativa } \\
+ \text { Cobertura relativa }) / 3)
\end{array}
$$

A partir de los datos de altura total, proyección de copas (X, Y), ubicación espacial de cada árbol, inclinación de la pendiente del terreno e IVF se generaron diagramas de perfil de la vegetación para las tres condiciones (FNA, B y FA). 
Tabla 1. Índices de diversidad alfa y beta calculados.

\begin{tabular}{lc}
\hline Diversidad alfa & $D_{M G}=\frac{(S-1)}{\ln (N)}$ \\
\hline Índice de Margalef & $H^{\prime}=\sum_{i=1}^{S} p_{i} \times \ln \left(p_{i}\right)$ \\
Índice de Shannon-Weiner & $D=\sum\left[\frac{n i(n i-1)}{N(N-1)}\right]$ \\
Índice de Simpson & $E=\frac{H^{\prime}}{\ln (S)}$ \\
\hline Equidad & $C_{S}(\%)=\left[\frac{2 j}{(a+b)}\right] \times 100$ \\
\hline Diversidad beta & $C_{j}(\%)=\left[\frac{j}{(a+b-j)}\right] \times 100$ \\
\hline Índice semejanza de Sorensen & \\
\hline Índice semejanza de Jaccard & \\
\hline
\end{tabular}

Donde: S: número de especies presentes, N: número total de individuos, ni: número de individuos de la especie $i$ y pi: proporción de individuos de la especie $i$, j: número de especie comunes en ambas comunidades, a: número de especies presentes en la comunidad a y b: número total de especies presentes en la comunidad b.

\section{Resultados}

\section{Composición florística}

En el área de estudio se registraron 1391 individuos agrupados en 15 especies arbóreas y 12 arbustivas, distribuidas en nueve familias (Tabla 2). Las FNA fueron las únicas zonas con presencia de especies únicas: Monnina ciliolata, Quercus candicans y Senecio roldana (Anexo 1).

\section{Curvas de acumulación especie-área}

De las cuatro curvas, solamente la del B (Fig 2b) no mostró una tendencia asintótica. En las curvas FNA (Fig. 2a), FA (Fig. 2c) y general (Fig. 2d) la tendencia asintótica de las curvas se alcanzó al cubrir un área de muestreo aproximada de $600 \mathrm{~m}^{2}, 400 \mathrm{~m}^{2}$ y $800 \mathrm{~m}^{2}$, respectivamente.

\section{Índices de diversidad}

Se registró una tendencia hacia una mayor riqueza (estimada por el índice de Margalef) en la FNA en comparación con las áreas de $\mathrm{B}$ y esta última mostró mayor riqueza que las FA; sin embargo, no hubo diferencias estadísticamente significativas entre las áreas en ninguno de los parámetros e índices calculados. Este mismo patrón se observó en la equidad específica (Tabla 3). En términos de diversidad, a pesar de no haber diferencias significativas entre las tres franjas, la FA mostró los valores menores de diversidad en ambos índices (Simpson y Shannon).

Las unidades de muestreo en la condición borde (B) presentaron mayor semejanza entre sí, con valores del coeficiente de Jaccard ( $\mathrm{Cj}$ ) entre 33\% y 89\%; y los del coeficiente de Sorensen (Cs) entre 53\% y 91\%. Los coeficientes obtenidos en las condiciones de franja no provechada (FNA) fueron Cj: $25 \%$ a $82 \%$ y Cs: $33 \%$ a $94 \%$ y para franja aprovechada (FA) fueron Cj: $20 \%$ a $86 \%$ y Cs: $55 \%$ a $91 \%$. 
TABLA 2. Listado de especies arbóreas y arbustivas presentes en el área silvícola de Ixtlán de Juárez.

\begin{tabular}{|c|c|c|}
\hline Familia & Nombre Científico & Nombre Común \\
\hline \multirow[t]{4}{*}{ Asteraceae } & Baccharis salicifolia (Ruiz y Pavón) Pers. & Chamizo \\
\hline & Telanthophora andrieuxii DC. & Bejuco de agua \\
\hline & Senecio roldana DC. & \\
\hline & Eupatorium lucidum Ort. & \\
\hline Betulaceae & Alnus acuminata Kunth. & Palo de águila \\
\hline \multirow[t]{2}{*}{ Ericaceae } & Arctostaphylos pungens H.B.K. & Pingüica \\
\hline & Arbutus xalapensis H.B.K. & Madroño \\
\hline \multirow[t]{9}{*}{ Fagaceae } & Calliandra grandiflora (L'Hér.) Benth. & Cabello de ángel \\
\hline & Quercus laurina Humb. y Bonpl. & Encino laurelillo \\
\hline & Quercus aff. laurina Humb. y Bonpl. & Encino \\
\hline & Quercus glaucoides Mart. y Gal. & Encino prieto \\
\hline & Quercus candicans Née & Encino ancho \\
\hline & Quercus castanea Née & Encino capulincillo \\
\hline & Quercus crassifolia Humb. y Bonpl. & Encino cucharilla \\
\hline & Quercus affinis Scheidw. & Encino \\
\hline & Quercus aristata Hook. y Arn. & Encino \\
\hline \multirow[t]{3}{*}{ Pinaceae } & Pinus pseudostrobus Lindl. & Pino liso \\
\hline & Pinus patula Schlecht. y Cham. & Pino colorado \\
\hline & Pinus leiophylla Schlecht. y Cham. & Pino \\
\hline Polygalaceae & Monnina ciliolata DC. & \\
\hline \multirow[t]{2}{*}{ Rhamnaceae } & Rhamnus sp. & \\
\hline & Rhamnus pompana M.C. Johnist. Et. L. A. & \\
\hline \multirow[t]{2}{*}{ Rosaceae } & Crataegus mexicana DC. & Tejocote \\
\hline & Prunus serotina Ehrh. & Cerezo de monte \\
\hline \multirow[t]{2}{*}{ Solanaceae } & Solanum cervantesii Lag. & \\
\hline & Cestrum sp. & \\
\hline
\end{tabular}

\section{Índices estructurales}

Setenta y seis punto cinco por ciento de IVI estuvo contenido en tres de las ocho familias registradas, Pinaceae (36.28\%), Fagaceae $(30.02 \%)$ y Solanaceae $(10.36 \%)$. Con base en el IVI, es posible distinguir semejanzas y diferencias en las tres condiciones evaluadas (FNA, B, FA) y entre los tres estados de desarrollo (fustal, latizal y brinzal; Tabla 4).
De acuerdo con el IVF las tres familias con mayor valor fueron: Fagaceae (51.06\%), Pinaceae (33.70\%) y Asteraceae $(5.72 \%)$, las cuales sumaron $90.48 \%$ del valor total forestal y $9.52 \%$ estuvo dividido entre las 5 familias restantes. En el IVF general, las especies con los más altos valores fueron Pinus pseudostrobus (33.71\%), Quercus crassifolia (28.68\%) y Quercus laurina (20.72\%) (Material suplementario). 

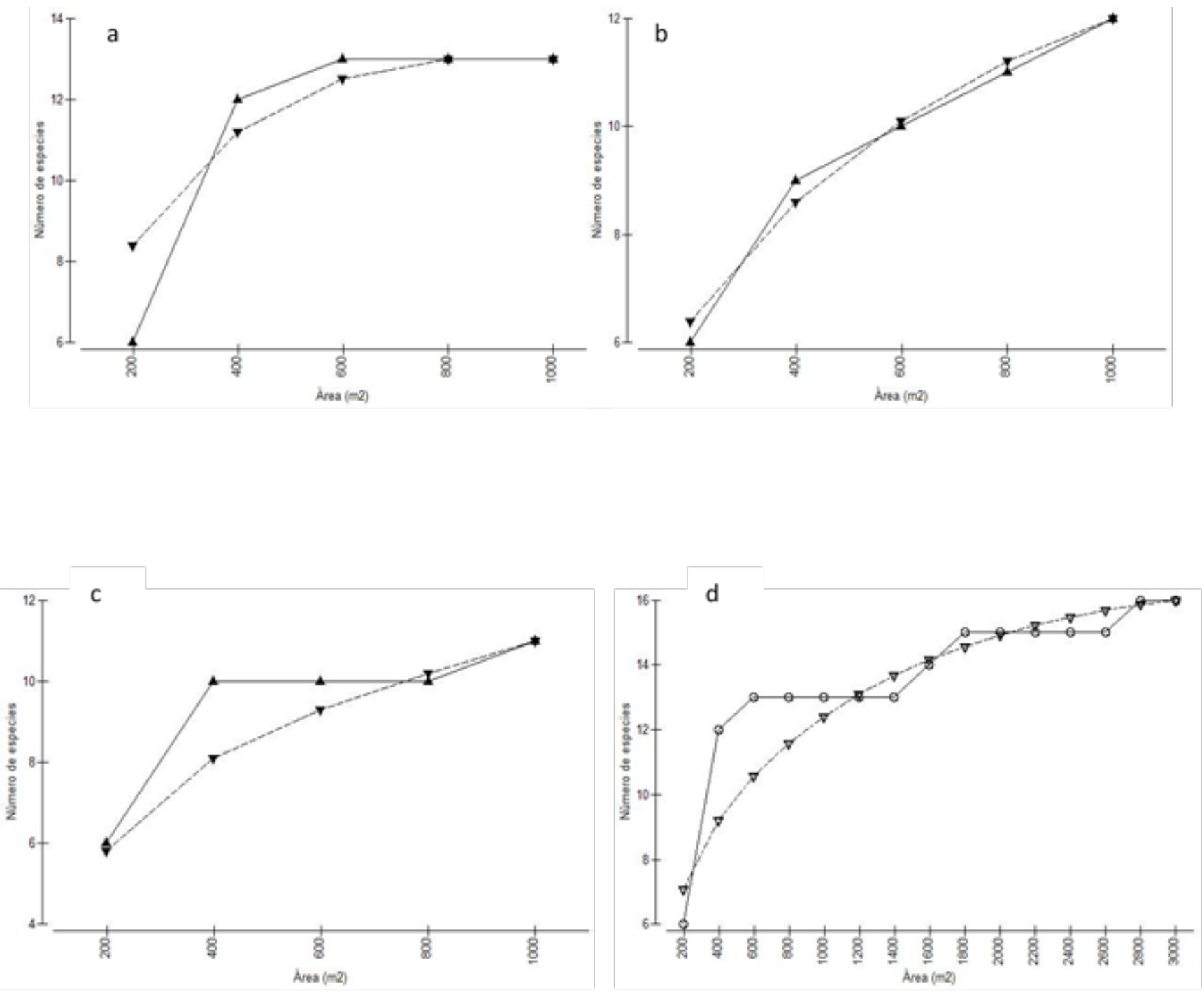

FIGURA 2. Curvas especie-área para las tres condiciones evaluadas: a) franjas no aprovechadas, b) borde, c) franjas aprovechadas y d) área total de muestreo.

TABLA 3. Abundancia y estimación de diversidad en las zonas evaluadas.

\begin{tabular}{lccc}
\hline & FNA & $B$ & FA \\
\hline No. de individuos & $495^{\mathrm{a}}$ & $475^{\mathrm{a}}$ & $421^{\mathrm{a}}$ \\
No. de Especies & $13^{\mathrm{a}}$ & $11^{\mathrm{a}}$ & $8^{\mathrm{a}}$ \\
Índice de Margalef & $1.63^{\mathrm{a}}$ & $1.41^{\mathrm{a}}$ & $1.05^{\mathrm{a}}$ \\
Índice de Shannon & $1.53^{\mathrm{a}}$ & $1.37^{\mathrm{a}}$ & $1.14^{\mathrm{a}}$ \\
Índice de Simpson & $0.28^{\mathrm{a}}$ & $0.33^{\mathrm{a}}$ & $0.41^{\mathrm{a}}$ \\
Equidad & $0.73^{\mathrm{a}}$ & $0.70^{\mathrm{a}}$ & $0.66^{\mathrm{a}}$ \\
\hline
\end{tabular}

FNA= franjas no aprovechadas; $B=$ borde; $F A=$ franjas aprovechadas.Medias seguidas por letra diferente entre columnas indican diferencias significativas (Tukey, $p<0.05, g l=2$ ). 
TABLA 4. Valores porcentuales del IVI en las principales especies arbóreas y arbustivas presentes en las franjas no aprovechadas (FNA), borde (B) y franjas aprovechadas (FA).

\begin{tabular}{cccccccccccc}
\hline Especie & \multicolumn{3}{c}{ Fustales } & \multicolumn{3}{c}{ Latizales } & \multicolumn{3}{c}{ Brinzales } \\
\hline & FNA & B & FA & FNA & B & FA & FNA & B & FA \\
\hline Q.cr & 29.74 & 20.48 & - & - & - & 6.44 & 10.63 & - & - \\
P.pse & 26.65 & 34.00 & - & - & 26.75 & 79.41 & - & 26.97 & 40.22 \\
Q.lau & 16.58 & 20.91 & - & - & 21.61 & 8.66 & 36.18 & 14.85 & - \\
Ces. & - & - & - & 40.53 & 20.61 & - & 25.65 & 23.52 & 13.60 \\
Bsal & - & - & - & 20.45 & - & - & - & - & 15.01 \\
M.cil & - & - & - & 8.01 & - & - & - & - & - \\
\hline
\end{tabular}

Q.cr = Quercus crassifolia; P.pse = Pinus pseudostrobus; Q.lau = Quercus laurina, Ce = Cestrum sp., B.sal = Baccharis salicifolia; M.cil = Monnina ciliolata

\section{DISCUSIÓN}

En los bosques de Ixtlán de Juárez, Oaxaca, la extracción maderera, principalmente de pinos, se hace a través de matarrasas por franjas alternas de acuerdo con el plan de manejo forestal elaborado en 2005 (TIASA, 2003). Este plan fue previamente certificado en 2001 (Smartwood, 2001), por el Consejo de Administración Forestal (FSC por sus siglas en ingles). Para la certificación, el FSC tomó en cuenta la aplicación de programas regulatorios, económicos y de mercado que promuevan el desarrollo, económico, social y ambiental.

Dentro del contexto ambiental, el FSC requiere anexar información sobre la biodiversidad presente y los mecanismos a seguir para su protección. En las áreas de bosque estudiadas, la biodiversidad arbórea y arbustiva está conformada por 27 especies, de las cuales ocho son endémicas de México (Pinus patula, Alnus acuminata, Telanthophora andrieuxii, Quercus laurina, Quercus afiinis, Quercus glaucoides, Pinus leiophylla y Crateagus mexicana) (García y Meave, 2011). Los resultados de diversidad alfa obtenidos en este estudio indican que 12 de las 15 unidades de muestreo tienen condiciones similares de vegetación. No obstante, sí se encontraron cambios en la composición arbórea y arbustiva. En FNA domina la presencia de especies arbustivas tolerantes a la sombra (Cestrum sp.,
Baccharis salicifolia) y una mezcla de especies arbóreas constituidas principalmente por encinos (e.g. Q. crassifolia y Q. laurina) y pinos (P. pseudostrobus). Las áreas B y FA, además de su alta semejanza florística (diversidad beta) debido a su continuidad y semejanza de condiciones ambientales (cfr. Luna, Almeida, Villers y Lorenzo, 1988), mostraron un estrato arbóreo dominado por $P$. pseudostrobus, que es la especie de interés comercial (RíosAltamirano et al., 2016), y un estrato dominado por plantas arbustivas heliófilas y de sucesión temprana.

La estimación de la estructura arbórea y arbustiva, estimada para cada condición a través del IVI y del IVF, está dominada por la presencia de Pinus pseudostrobus, Quercus laurina y Quercus crassifolia. Esta última es la especie dominante en la condición FNA, mientras que $P$. pseudostrobus domina en las condiciones B y FA. De las especies mencionadas, $P$. pseudostrobus es heliófita y las condiciones B y FA permiten el desarrollo de esta especie al ser sitios con mayor disponibilidad de luz. Además, $P$. pseudostrobus junto con $P$. patula, son las especies comúnmente utilizadas en el programa de reforestación aplicado en las zonas manejadas (Ríos-Altamirano et al., 2016). Para brinzales en la condición FNA las especies dominantes fueron $Q$. laurina y Cestrum sp., mientras que $P$. psendostrobus fue la especie dominante en las condiciones 
B y FA. Cabe resaltar que las especies arbóreas presentes en el estado de desarrollo brinzal y latizal coinciden tanto en las FA como en las FNA, lo que sugiere que el tipo de manejo no provoca un cambio permanente en su riqueza arbórea, como también ha sido descrito en otros estudios (e.g. Chaudhary, Burivalova, Koh y Hellweg, 2016; Edwards, Tobias, Sheil, Meijaard y Laurance, 2014).

Los índices de diversidad tanto de Jaccard como de Sorensen (diversidad beta; Tabla 1) indican que las condiciones $\mathrm{B}$ y FA son las de mayor semejanza florística. Ambas condiciones se encuentran contiguas y cuentan con mayor disponibilidad de luz como resultado de la apertura de claros por el aprovechamiento forestal. Luna, Almeida, Villers y Lorenzo (1988) mencionan que las zonas más cercanas tienen mayor semejanza florística. A pesar de que FNA y B son contiguas, son también las áreas que tuvieron la menor semejanza, algo obvio al considerar que la matarrasa tiende a generar una modificación extrema de la estructura original del bosque.

La composición florística, expresada a partir de los diagramas de perfil vertical (Material suplementario) para latizales en las tres condiciones (FNA, B y FA) está conformada por la presencia de tres especies: Cestrum sp., Baccharis salicifolia y Pinus pseudostrobus. Tanto Cestrum sp., como B. salicifolia son especies arbustivas tolerantes a la sombra y se presentaron con mayor abundancia en FNA, bajo el dosel de los fustales, donde no se presentó extracción de individuos. P. pseudostrobus es una especie arbórea común en FA, esta especie se desarrolla adecuadamente en condiciones de mayor luminosidad, principalmente donde hay claros amplios como los generados en las FA y en B (Challenger, 1998).

De acuerdo con diversos autores, el proceso de regeneración que se promueve en las matarrasas favorece el establecimiento de bosques coetáneos dominados por pino, este mecanismo puede ser un promotor de disminución de la biodiversidad al eliminar a las especies poco tolerantes a la perturbación, además de alterar los procesos funcionales de este ecosistema (Chaudhary, Burivalova, Koh y Hellweg, 2016; Ishii, Tanabe y Hiura, 2004; Spiecker, 2003; Thompson, Baker, Ter-Mikaelian, 2003). Es probable que en las franjas de extracción forestal se hayan presentado una serie de cambios tanto físicos como biológicos a consecuencia de los aprovechamientos, los cuales permiten una rápida germinación de semillas de especies efímeras o pioneras (e.g. Baccharis salicifolia., Cestrum sp., Arbutus xalapensis, Alnus acuminata, entre otras). Un mecanismo tendiente a mitigar los efectos adversos de este tipo de manejo son las FNA, estas franjas permiten una rápida colonización y tienden a disminuir o impedir la pérdida definitiva de diversidad en las zonas bajo aprovechamiento (Finegan 1984; Drury y Nisbet 1973) (Material suplementario).

A pesar de que existe extracción forestal y que esta tiende a modificar la estructura del bosque, su diversidad arbórea y arbustiva es propia de un bosque natural; por lo tanto, a corto plazo las especies presentes en las áreas de FA y B siguen la misma tendencia sucesional encontrada en las FNA. Por otro lado, la perturbación, causada por el proceso de extracción maderable influye de manera diferencial en los patrones y procesos de este ecosistema (Puettmann, Coates y Messier, 2008). La permanencia de los servicios ecosistémicos originales no se puede asegurar en el bosque aprovechado, de acuerdo con Llano y Fernández (2017) y Thompson et al. (2011). En áreas forestales aprovechadas de Ixtlán de Juárez, se han detectado modificaciones en la estructura de la vegetación (Zacarías-Eslava y Del Castillo 2010; Castellanos et al., 2008) y cambios en las propiedades físicas y químicas del suelo (Zaragoza-López, 2016), los cuales pueden agravarse al crear condiciones uniformes en estructura y composición (Puettmann, Coates y Messier, 2008). Por este motivo, el mitigar los cambios en la estructura original de los bosques de Ixtlán de Juárez es importante, dado que esta área se encuentra situada en una de las zonas terrestres prioritarias del país.

Es necesario tomar en cuenta todo el conjunto de información presente y considerar los posibles cambios estructurales generados durante el proceso de manejo forestal. Para que el plan de manejo implementado por Servicios Técnicos Forestales (STF, 2014) logre su meta de extracción maderera, sin deteriorar la dinámica de este 
ecosistema, es necesario considerar la información generada en la actualidad, de tal manera que sea factible replantear mecanismos de manejo en tiempo real. Por ejemplo, a pesar de que las tres condiciones son contiguas, cada una presentó un patrón estructural propio, por lo que es necesario un monitoreo flexible, a partir de considerar los atributos estructurales de las zonas tanto previo al manejo, como durante los procesos sucesionales posteriores al proceso extractivo. Todavía es necesario seguir vinculado esfuerzos interdisciplinarios para lograr generar procesos de manejo sin menoscabar la estructura, composición y diversidad en las áreas de aprovechamiento forestal.

\section{CONCLUSIONES}

La presente investigación muestra que el tipo de manejo para el periodo analizado no provoca una pérdida de especies arbóreas y arbustivas, pero sí genera un cambio estructural importante, además de tender a favorecer la presencia de bosque con una estructura homogénea a corto plazo, pero con la tendencia a una estructura heterogenea conforme los individuos de las especies arbóreas en las categorías latizales y brinzales van desarrollándose. Ahora bien, a diferencia de bosques naturales, la estructura que se tiende a favorecer estará asociada al desarrollo de las especies de Pinus, en particular Pinus pseudostrobus. Si se busca promover planes de manejo que permitan continuar con una estructura boscosa cercana a la natural y evitar la pérdida de la diversidad biológica, se tiene que considerar continuar con el monitoreo de las comunidades vegetales, sin descuidar los estudios de las comunidades de animales y de las condiciones físicas y biológicas del suelo, por mencionar algunos; de tal manera que puedan realizarse los ajustes necesarios a los planes de manejo previamente certificados.

\section{RECONOCIMIENTOS}

Se agradece a PROMEP (UNSIJ-PTC-001, convocatoria 2011) por los recursos económicos otorgados a través del proyecto "Efecto que ejerce el manejo silvícola sobre las especies de anfibios y reptiles en los bosques de Ixtlán de Juárez, Oaxaca". VAH agradece al Posgrado en Ciencias Biológicas y Conacyt, por el apoyo otorgado durante la estancia sabática y RCT agradece el apoyo otorgado por Conacyt a través del proyecto SEP-Conacyt 180790.

\section{REFERENCIAS}

Alfonso-Corrado, C., Pérez-Legaspi, A., Campos-Contreras, J., Gorgonio-Ramírez, M., Velasco-Hipólito, F., \& ClarkTapia, R. (2016). Región hidrológica 28: Cuenca del Papaloapan. En: R. Clark-Tapia, M. F. Ramos-Morales, C. Alfonso-Corrado, M. M. Mendoza-Díaz y M. F. RamosMorales (Eds.). Recursos Hidricos de la Sierra Norte de Oaxaca: Caracterización, Diagnóstico y Gestión (pp. 12-23). México: Universidad de la Sierra Juárez.

Arriaga, L., Espinoza, J. M., Aguilar, C., Martínez, E., Gómez, L., \& Loa, E. (Coords.) (2000). Regiones terrestres prioritarias de México. México: Comisión Nacional para el Conocimiento y uso de la Biodiversidad (Conabio).

Castellanos-Bolaños, J. F., Treviño-Garza, E. J., AguirreCalderón, O. A., Jiménez-Pérez, J., \& Velázquez-Martínez, A. (2010). Diversidad arbórea y estructura espacial de bosques de pino-encino en Ixtlán de Juárez, Oaxaca. Revista Mexicana de Ciencias Forestales, 1(2), 39-52.

Castellanos-Bolaños. J. F., Treviño-Garza, E. J., AguirreCalderón O. A., Jiménez-Pérez, J. Musálem-Santiago, M., \& López-Aguillón, R. (2008). Estructura de bosques de Pinus patula bajo manejo en Ixtlán de Juárez, Oaxaca, México. Madera y Bosques, 14(2), 51-63. doi: 10.21829/myb.2008.1421212

Challenger, A. (1998). Utilización y conservación de los ecosistemas terrestres de México: pasado, presente y futuro. México: Conabio, Instituto de Biología de la UNAM y Agrupación Sierra Madre S. C.

Chaudhary, A., Burivalova, Z., Koh, L. P., \& Hellweg, S. (2016). Impact of forest management on species richness: global meta-analysis and economic trade-offs. Scientific reports, 6(23954). doi:10.1038/srep23954.

Clark-Tapia, R., Fuente-Carrasco, M., Alfonso-Corrado, C., Ramos-Morales, M. F., \& Aguirre-Hidalgo, V. 2017. Manejo forestal comunitario y sustentabilidad en Sierra Juárez, Oaxaca. Mexico: Fontamara.

Clark-Tapia, R., Rodríguez-Rivera, V., Ramos-Morales M. F., \& Velasco-Hipólito, F. (2016). Tipos de suelos. En R. ClarkTapia, M. F. Ramos-Morales, C. Alfonso-Corrado, M. M. 
Mendoza-Díaz, \& M. F. Ramos-Morales (Eds.). Recursos Hidricos de la Sierra Norte de Oaxaca: Caracterización, Diagnóstico y Gestión (pp. 35-42). México: Universidad de la Sierra Juárez.

Clark-Tapia, R., Suárez-Mota, M. E., Venegas-Barrera, C. S., \& Velasco-Hipólito, F. (2016). Clima. En R. Clark-Tapia, M. F. Ramos-Morales, C. Alfonso-Corrado, M. M. MendozaDíaz, \& M. F. Ramos-Morales (Eds.). Recursos hidricos de la Sierra Norte de Oaxaca: caracterización, diagnóstico y gestión (pp. 43-47). México: Universidad de la Sierra Juárez.

Clark-Tapia, R., Suárez-Mota, M. E., Matías-Nolasco, M., \& Velasco-Hipólito, F. (2016). Clima: pasado, presente y futuro. En R. Clark-Tapia, M. F. Ramos-Morales, C. Alfonso-Corrado, M. M. Mendoza-Díaz, \& M. F. RamosMorales (Eds.). Recursos bidricos de la Sierra Norte de Oaxaca: caracterización, diagnóstico y gestión (pp. 48-57). México: Universidad de la Sierra Juárez.

Clark-Tapia, R., Sandoval-García, R., Ramos-Morales, M. F., \& Rodríguez-Rivera, V. (2016). Cambio de uso de suelo y cobertura vegetal. En R. Clark-Tapia, M. F. RamosMorales, C. Alfonso-Corrado, M. M. Mendoza-Díaz, \& M. F. Ramos-Morales (Eds.). Recursos hidricos de la Sierra Norte de Oaxaca: caracterización, diagnóstico y gestión (pp. 65-75). México: Universidad de la Sierra Juárez.

Clarke, K. R., \& Gorley R. N. (2006). PRIMER v6: user manual/tutorial. PRIMER-E, UK: Plymouth.

Corral-Rivas, J. Jiménez-Pérez, J., Aguirre-Calderón, O. A., \& Corral-Rivas, S. (2005). Un análisis del efecto del aprovechamiento forestal sobre la diversidad estructural en el bosque mesófilo de montaña "El Cielo". Investigación Agraria: Sistemas y Recursos Forestales, 14(1), 217-228.

Luteyn L. J., Clemants, S. E., Diggs, G. M., Dorr, L. J., Judd, W. S., Luteyn, J. L., Sorenson, P. D., Stevens, P. F., \& Wallace, G. D. (1995). ERICACEAE Part II the superior-ovaried genera Monotropoideae, Pyroloideae, Rhododendroideae and V accinioideae p.p). Flora Neotropica Monograph 66. Estados Unidos: The New York Botanical Garden Press.

Comisión Nacional Forestal [Conafor]. (2008). Programa estratégico forestal del estado de Oaxaca, 2007-2030. México: Conafor.

Corella-Justavino, F., Valdez-Hernández, J. I., Cetina-Alcalá, V. M., González-Cossio, F. V., Trinidad-Santos, A., \& Aguirre-Rivera, J. R. (2001). Estructura forestal de un bosque de mangles en el noreste del estado de Tabasco, México. Ciencia Forestal en México, 26(90), 73-102.
Curtis, J. T, \& McIntosh, R. P. (1951). An upland forest continuum in the pariré-forest border region of Wisconsin. Ecology, 32(3), 476-496. doi: 10.2307/1931725.

Del Castillo, R. F., Pérez de la Rosa, J. A., Vargas, A., \& Rivera, R. (2004). Coníferas. En A. J. García-Mendoza; M. Ordóñez, \& M. Briones (Eds.), Biodiversidad de Oaxaca (pp. 141-158). México: Instituto de Biología, UNAM-Fondo Oaxaqueño para la Conservación de la Naturaleza-WWF.

Drury, W. H., \& Nisbet, I. C. T. (1973). Succession. Journal of the Arnold Arboretum, 54(3), 331-368.

Edwards, D. P., Tobias, J. A., Sheil, D., Meijaard, E., \& Laurance, W. F. 2014. Maintaining ecosystem function and services in logged forests. Trends in Ecology and Evolution, 29(9), 511520. doi: 10.1016/j.tree.2014.07.003.

Farjon, A., Pérez de la Rosa J. A., \& Styles, B. T. (1997). Guía de campo de los pinos de México y América Central. England: The Royal Botanic Gardens, Kew.

Finegan, B. (1984). Forest succession. Nature, 312, 109-114. doi:10.1038/312109a0

Fregoso, A., Velásquez, A., Brocco, G., \& Cortéz, G. (2001). El enfoque de paisaje en el manejo forestal de la comunidad indígena de Nuevo San Juan Parangaricutiro, Michoacán, México. Investigaciones Geográficas. Boletin del Instituto de Geografia, UNAM, 46, 58-77.

García-Mendoza, A. J., \& Meave del Castillo J. A. (2011). Diversidad florística de Oaxaca: de musgos a angiospermas. (Colecciones y lista de especies). México: UNAM-Conabio.

Hawley, R. C., \& Smith, D. M. (1982). Silvicultura práctica. Barcelona: Ediciones Omega.

Ishii, H. T., Tanabe, S-I., \& Hiura, T. (2004). Exploring the relationships among canopy structure, stand productivity, and biodiversity of temperate forest ecosystems. Forest Science, 50(3), 342-355.

Jiménez, J., Aguirre, O., \& Kramer, H. (2001). Análisis de la estructura horizontal $y$ vertical en un ecosistema multicohortal de pino-encino en el norte de México. Investigaciones Agrarias Sistema de Recursos Forestales, 10(2), $355-366$.

Koleff, P., Gaston, K. J., \& Lennon, J. J. (2003). Measuring beta diversity for presence-absence data. Journal of Animal Ecology, 72(3), 367-382. doi: 10.1046/j.13652656.2003.00710.x

Luna, I., Almeida, L., Villers, L., \& Lorenzo, L. (1988). Reconocimiento florístico y consideraciones 
fitogeográficas del bosque mesófilo de montaña de Teocelo, Veracruz. Boletín de la Sociedad Botánica de México, 48, 35-63.

Llano, Manuel, \& Fernández, H. (Comps). 2017. Análisis y propuestas para la conservación de la biodiversidad en México 1995. 2017. México.

Margalef, R. (1977). Ecología, Barcelona: Omega ediciones.

McVaugh, R. (1984). Flora Novo-Galiciana: a descriptive account of the vascular plants of western México. Vol. 12. Compositae. Estados Unidos: The University of Michigan Press.

Murcia, C. (1995). Edge effects in fragmented forests: implications for conservation. Trends in Ecology and Evolution, 10(2), 58-62. doi: 10.1016/S0169-5347(00)889776.

Puettmann, K. J., Coates, K. D., \& Messier, C. (2008). A critique of silviculture: Managing for complexity. Estados Unidos: Island Press.

Ríos-Altamirano, A., Alfonso-Corrado, C., Aguirre-Hidalgo, V., Ángeles-Pérez, G., Mendoza-Díaz, M. M., RodríguezRivera, V., Roldán-Félix, E., \& Clark-Tapia, R. (2016). Abundancia y distribución del género Pinus en Capulálpam de Méndez, Sierra Juárez, Oaxaca. Madera y Bosques, 22(3), 61-74. doi:10.21829/myb.2016.2231457.

Ruiz-Aquino, F., Valdez-Hernández, J. I., Manzano-Méndez, F., Rodríguez-Ortiz, G., Romero-Manzanares, A., \& FuentesLópez, M. E. (2014). Ecuaciones de biomasa aérea para Quercus laurina y Q. crassifolia en Oaxaca, México. Madera y Bosques, 20(2), 33-48. doi:10.21829/myb.2014.202162.

Rzedowski, G. C. de, Rzedowski, J., \& colaboradores. (2005). Flora fanerogámica del Valle de México. (2a ed.). 1a reimp. México: Instituto de Ecología, A.C., Conabio.

Secretaria de Medio Ambiente y Recursos Naturales [Semarnat] (2012). Programa sectorial de medio ambiente y recursos naturales (PROMARNAT) 2013-2018. México: Diario Oficial de la Federación 12 de diciembre de 2013.

Servicios Técnicos Forestales de Ixtlán de Juárez (STF). (2014). Programa de manejo forestal para el aprovechamiento y conservación de los recursos forestales maderables de Ixtlán de Juárez: Ciclo de corta 2015-2024.

Smartwood. (2001). Resumen Público de Certificación de la Comunidad de Ixtlán de Juárez. Recuperado de https://www.rainforest-

alliance.org/business/forestry/documents/ixtlan_juarez.p df
Spiecker, H. (2003). Silvicultural management in maintaining biodiversity and resistance of forest in Europe-temperate zone. Journal of Evolutionary Biology, 67(1), 55-65. doi.org/10.1016/S0301-4797(02)00188-3.

Técnica Informática Aplicada S.A., (TIASA). (2003). Programa de manejo forestal para el aprovechamiento persistente de los recursos naturales forestales maderables ciclo 2003-2013, para la comunidad de Ixtlán de Juárez, Oaxaca., México.

Thompson, I. D., Okabe, K., Tylianakis, J. M., Kumar, P., Brockerhoff, E. G., Schellhorn, N. A., Parrota, J. A., \& Nasi, R. (2011). Forest biodiversity and the delivery of ecosystem goods and services: Translating science into policy. BioScience, 61(12), 972-981. doi.org/10.1525/bio.2011.61.12.7.

Thompson, I. D., Baker J. A., Ter-Mikaelian, M. (2003). A review of the long-term effects of post-harvest silviculture on vertebrate wildlife, and predictive models, with emphasis on boreal forest in Ontario, Canada. Forest Ecology and Management, 177(1-3), 441-469. doi.org/10.1016/S03781127(02)00453-X.

Valdez-Hernández, J. I. (2002). Aprovechamiento forestal de manglares en el estado de Nayarit, costa Pacífica de México. Madera y Bosques, 8: 129-145.

Valencia-Avalos, S., \& Nixon, K. C. (2004). Encinos. En: A. J. García-Mendoza, M. J. Ordóñez, \& M. Briones-Salas (Eds.). Biodiversidad de Oaxaca (pp. 219-225). México: Instituto de Biología-UNAM, Fondo Oaxaqueño para la Conservación de la Naturaleza, World Wildlife Fund.

Zaragoza-López, K. (2016). Caracterización de suelos bajo manejo forestal en Ixtlán de Juárez, Oaxaca (Tesis de Licenciatura). Universidad de la Sierra Juárez, Oaxaca, México.

Zacarías-Eslava, Y., \& Del Castillo, R. F. (2010). Comunidades vegetales de la Sierra Juárez, Oaxaca: pisos altitudinales y sus posibles implicaciones ante el cambio climático. Boletín de la Sociedad Botánica Mexicana, 87: 1328.

Zavala, C. F. (2003). Identificación de encinos de México. (2a ed.). México: Universidad Autónoma de Chapingo, División de Ciencias Forestales.

Manuscrito recibido el 10 de octubre de 2017

Aceptado el 27 de abril de 2018

Publicado el 26 de noviembre de 2018 
Este documento se debe citar como:

Vásquez-Cortez, V. F., Clark-Tapia, R., Manzano-Méndez, F., González-Adame, G., \& Aguirre-Hidalgo. V. (2018). Estructura, composición y diversidad arbórea y arbustiva en tres condiciones de manejo forestal de Ixtlán de Juárez, Oaxaca. Madera y Bosques, 24(3), e2431649. doi:

Madera y Bosques por Instituto de Ecología, A.C. se distribuye bajo una Licencia Creative Commons Compartirlgual 4.0 Internacional.

10.21829/myb.2018.2431649

ANEXO 1. Listado de especies arbóreas y arbustivas presentes en los sitios por área de manejo forestal.

\begin{tabular}{|c|c|c|c|}
\hline ESPECIE & FNA & $\mathrm{B}$ & FA \\
\hline Alnus acuminata. & 1 & 1 & 1 \\
\hline Arbutus xalapensis & 1 & 1 & 1 \\
\hline Baccharis salicifolia & 1 & 1 & 1 \\
\hline Cestrum sp. & 1 & 1 & 1 \\
\hline Eupatorium lucidum & 1 & 1 & 0 \\
\hline Monnina ciliolata & 1 & 0 & 0 \\
\hline Pinus pseudostrobus & 1 & 1 & 1 \\
\hline Pinus patula & 0 & 1 & $\mathrm{O}$ \\
\hline Prunus serotina & 1 & 1 & 1 \\
\hline Quercus crassifolia & 1 & 1 & 1 \\
\hline Quercus castanea & 0 & 1 & 0 \\
\hline Quercus candicans & 1 & 0 & 0 \\
\hline Quercus laurina & 1 & 1 & 1 \\
\hline Senecio roldana & 1 & 0 & 0 \\
\hline Telanthophora andrieuxii & 1 & $\mathrm{O}$ & $\mathrm{O}$ \\
\hline
\end{tabular}

FNA = franja no aprovechada, $\mathrm{B}=$ borde, $\mathrm{FA}$ = franja aprovechada, $\mathrm{AT}$ = área total 1 = presente, $\mathrm{O}=$ ausente 